Pacific Journal of Mathematics

MAPS ON SIMPLE ALGEBRAS PRESERVING ZERO
PRODUCTS. I. THE ASSOCIATIVE CASE 


\title{
MAPS ON SIMPLE ALGEBRAS PRESERVING ZERO PRODUCTS. I: THE ASSOCIATIVE CASE
}

\author{
W. J. WONG
}

\begin{abstract}
Recent studies of linear transformations of various types on the space of $n \times n$ matrices over a field suggest the general problem of finding the semilinear transformations $f$ on an algebra $A$ over a field $k$, with the property that
\end{abstract}

$$
x y=0 \Longrightarrow f(x) f(y)=0,
$$

where $x, y \in A$. In this article such maps are determined for a class of primitive associative algebras, including the case of bijective maps $f$ on a finite-dimensional simple associative algebra $A$.

Introduction. In recent years there have been many investigations characterizing the linear transformations on the $n \times n$ matrix algebra $M_{n}(k)$ over a field $k$ which preserve various properties, one of the earliest being a theorem of Dieudonne finding the bijective linear maps preserving the set of singular matrices in $M_{n}(k)$ [4], with other studies concerning maps preserving rank, various algebraic groups, etc. $[1,2,5,6,8]$. In many cases, the problems considered can be easily formulated entirely in terms of the structure of $M_{n}(k)$ as an associative or Lie algebra, and can therefore be investigated in other algebras. For example, a theorem of Watkins [9] finds the bijective linear transformations $f$ on $M_{n}(k)$ satisfying the condition that $[f(x), f(y)]=0$ for all pairs of elements $x, y$ of $M_{n}(k)$ such that $[x, y]=0$, where [,] denotes the usual Lie product in $M_{n}(k)$. This naturally suggests that the same problem be investigated for other algebras. If $A$ is any algebra (not necessarily an associative or Lie algebra) over a field $k$, we can seek to determine the set $G(A)$ of all bijective linear transformations $f$ on $A$ with the property that $f(x) f(y)=0$ for all pairs of elements $x, y$ of $A$ such that $x y=0$. We say that such a map $f$ preserves zero products.

If $A$ is finite-dimensional, the set of all ordered pairs $x, y$ for which $x y=0$ is an algebraic set in the affine space $A \times A$, and an easy modification of the proof of a lemma of Dixon [5, p. 386] shows that $G(A)$ is a group. Clearly $G(A)$ contains the automorphism group $G_{1}$ of $A$, the group of units $G_{2}$ of the centroid of $A$ (the algebra of linear transformations which commute with both left and right multiplications in $A$ ), and the group $G_{3}$ of all bijective transformations $f$ of the form $f(x)=x+g(x)$, where $g$ is a linear map of $A$ into the ideal $A^{0}$ of all elements $z$ for which $z A=A z=$ 0 . The product $G_{0}(A)=G_{1} G_{2} G_{3}$ is a subgroup of $G(A)$. (If $A^{0}=0$, 
then $G_{2}$ is abelian and $G_{0}(A)$ is the semidirect product of $G_{1}$ and $G_{2}$ [7, p. 107]).

Following Dieudonné [4], we can generalize our problem slightly by taking for $G(A)$ the set of all bijective semilinear maps $f$ on $A$ which preserve zero products, and enlarging $G_{0}(A)$ by taking $G_{1}$ to be the group of all semilinear automorphisms of $A$ (semilinear maps which are automorphisms of the multiplicative structure). We can also consider more general situations in which $A$ may not be finitedimensional, or the maps $f$ are not assumed to be bijective. Since there is no problem if all products in $A$ are zero (so that all subspaces are ideals), we shall examine the opposite case, where $A$ is simple or nearly simple. Of particular interest will be classical algebras of linear transformations, for which our problem is somewhat analogous to the problem of finding the automorphisms of the classical groups [3].

We deal first with the case that $A$ is a simple associative algebra, or more generally a primitive associative algebra. If $A$ is assumed to possess minimal left or right ideals, there is a natural topology defined on $A$, and also an associated division algebra $D$. Under the hypothesis that $D$ is finite-dimensional over $k$, we show that, except in the trivial case that $A$ is a division algebra, a continuous semilinear transformation which preserves zero products and satisfies a condition weaker than bijectivity must essentially lie in $G_{0}(A)$ (Theorem $\mathrm{C}$ and Corollary D). In particular, if $A$ is finitedimensional, so that the topology is discrete, we have $G(A)=G_{0}(A)$.

The solution of this problem is obtained by applying a result concerning maps on tensor products of vector spaces over division algebras, which preserve elements of rank 1 . This situation has been considered by a number of authors, e.g., Jacob [6], Marcus and Moyls [8], but the exact result we need (Theorem A) does not seem to be in the literature. With some variations and complications, the methods of this article will also play an essential role in the case of nonassociative simple algebras which we shall consider in subsequent papers. For example, a study of "generalized rank 1 preservers" will be used in [10] to determine maps preserving zero products for a class of simple Lie algebras constructed from the finite-dimensional simple associative algebras.

Since the transformations occurring in the conclusion of Theorem $\mathrm{C}$ and Corollary $\mathrm{D}$ are bijective, the hypotheses of these results may also be regarded as providing some information about maps preserving zero products which are not bijective. The last section of the paper gives some examples of such maps. It remains an open problem to determine the maps preserving zero products which do not satisfy our conditions, although a recent paper of Botta throws 
some light on the question when $A$ is finite-dimensional and $k$ is algebraically closed [1].

1. Maps on tensor products. We first recall the definition and some basic facts about tensor products of vector spaces over a division ring. Suppose $D$ is a division ring, $U$ is a right vector space over $D$, and $V$ is a left vector space over $D$. If $H$ is any additive abelian group, a map $b: U \times V \rightarrow H$ is said to be balanced if it is additive in each variable and $b(u \alpha, v)=b(u, \alpha v)$, for all $u \epsilon$ $U, v \in V, \alpha \in D$. A tensor product of $U$ and $V$ consists of an additive abelian group $T$ with a balanced map $t: U \times V \rightarrow T$, with the property that every balanced map $b: U \times V \rightarrow H$ can be factored uniquely, $b=g t$, where $g$ is a homomorphism of $T$ into $H$. Then $T$ exists and is essentially unique. Usually $T$ is denoted $U \otimes V$ and, if $u \in U, v \in V, t(u, v)$ is denoted $u \otimes v$. An element $x$ of $U \otimes V$ is said to be of rank 1 if $x=u \otimes v$, where $u$ and $v$ are nonzero. A rank 1 subgroup of $U \otimes V$ is a subgroup whose nonzero elements are all of rank 1. The elements of rank 1 generate $U \otimes V$. In an expression of an element $x$ of rank 1 in the form $x=u \otimes v$, the subspaces $\langle u\rangle,\langle v\rangle$ generated by $u$ and $v$ are uniquely determined, and will be denoted $U(x), V(x)$.

Lemma 1. If $x, y$ are elements of rank 1 in $U \otimes V$ such that $x+y$ is either of rank 1 or zero, then $U(x)=U(y)$ or $V(x)=V(y)$.

Proof. Any element $p$ of the dual space $U^{*}$ of $U$ induces a homomorphism $\bar{p}: U \otimes V \rightarrow V$ such that $\bar{p}(u \otimes v)=p(u) v$, and so $\bar{p}(u \otimes v) \in V(u \otimes v)$.

Suppose that $x=u \otimes v, y=u_{1} \otimes v_{1}$, and assume that $U(x) \neq U(y)$. Then there exist $p, q$ in $U^{*}$ such that

$$
p(u)=1, p\left(u_{1}\right)=0, q(u)=0, q\left(u_{1}\right)=1 .
$$

Then $\bar{p}(x+y)=\bar{p}(x)+\bar{p}(y)=v, \bar{q}(x+y)=\bar{q}(x)+\bar{q}(y)=v_{1}$. Hence $v$ and $v_{1}$ lie in $V(x+y)$, so that $V(x)=V(x+y)=V(y)$.

For convenience we write $U^{\sharp}, V^{\sharp}$ for the sets of nonzero elements of $U, V$ respectively. If $u \in U^{\sharp}$, the set

$$
u \otimes V=\{u \otimes v \mid v \in V\}
$$

is a rank 1 subgroup of $U \otimes V$. Similarly, an element $v$ of $V^{\sharp}$ determines a rank 1 subgroup

$$
U \otimes v=\{u \otimes v \mid u \in U\}
$$


LEMma 2. If $H$ is a rank 1 subgroup of $U \otimes V$, then either $H \subseteq u \otimes V$, some $u \in U^{\sharp}$, or $H \leqq U \otimes v$, some $v \in V^{\sharp}$.

Proof. Assume that $H$ is not contained in $u \otimes V$ for any $u$. Then there exist nonzero $x, y$ in $H$ such that $U(x) \neq U(y)$. By Lemma $1, V(x)=V(y)$. If $z$ is any nonzero element of $H$, then either $U(\boldsymbol{z}) \neq U(x)$ or $U(z) \neq U(y)$. By Lemma $1, V(z)=V(x)$ or $V(z)=V(y)$. In any case, $V(z)=V(x)$, and so $H \subseteq U \otimes v$, where $V(x)=\langle v\rangle$.

If $D$ is a division algebra over a field $k$, which may be identified with a subfield of the center of $D$, then $U \otimes V$ can be made into a vector space over $k$, with scalar multiplication such that $c(u \otimes v)=u \otimes(c v)$, where $u \in U, v \in V, c \in k$. If $u \in U^{\sharp}$, then $u \otimes V$ is a subspace of the $k$-vector space $U \otimes V$, and the map $v \rightarrow u \otimes v$ is an isomorphism of $V$ with $u \otimes V$ as $k$-vector spaces. Similarly, if $v \in V^{\sharp}, U \otimes v$ is a subspace of $U \otimes V$ isomorphic with $U$.

Before stating the main result of this section, we recall that a map $h$ of $V$ into another left vector space $V_{1}$ over $D$ is said to be semilinear with respect to an automorphism $\sigma$ of $D$ (more briefly, $\sigma$-semilinear) if $h$ is additive and $h(\alpha v)=\alpha^{\sigma} h(v)$, for all $v \in V, \alpha \in D$. Similarly one can define semilinear maps between right vector spaces. Also, semilinear maps can be defined from a left vector space to a right vector space, or vice versa, with respect to an anti-automorphism of $D$. Further, if $V$ and $V_{1}$ are left vector spaces over a division algebra $D$ over a field $k$, then the maps of $V$ into $V_{1}$ (regarded as vector spaces over $k$ ) which are semilinear with respect to a given automorphism $\mu$ of $k$ form a right vector space $L_{k}\left(V, V_{j}\right)$ over $D$, with the product $h \alpha$ of an element $h$ of $L_{u}\left(V, V_{1}\right)$ by an element $\alpha$ of $D$ being given by the equation

$$
(h \alpha)(v)=h(\alpha v),
$$

where $v \in V$. Similarly, if $U, U_{1}$ are right vector spaces over $D$, the $\mu$-semilinear maps of $U$ into $U_{1}$ form a left vector space $L_{: \prime}(U$, $U_{1}$ ) over $D$.

THEOREM A. Let $D$ be a finite-dimensional division algebra over a field $k$, let $U, U_{1}$ be right vector spaces over $D$, let $V, V_{1}$ be left vector spaces over $D$, and suppose $\operatorname{dim}_{D} U>1, \operatorname{dim}_{D} V>1$. If $f: U \otimes V \rightarrow U_{1} \otimes V_{1}$ is a semilinear map with respect to an automorphism $\mu$ of $k$, and $f$ maps rank 1 elements on rank 1 elements, then one of the following holds.

(i) There exists a nonzero element $u_{1}$ of $U_{1}$ and a D-linear. map $u \rightarrow h_{u}$ of $U$ into $L_{u}\left(V, V_{1}\right)$, such that $h_{u}$ is injective for all nonzero $u$ in $U$, and 


$$
f(u \otimes v)=u_{1} \otimes h_{u}(v),
$$

for all $u \in U, v \in V$.

(ii) There exists a nonzero element $v_{1}$ of $V_{1}$ and a D-linear. map $v \rightarrow g_{v}$ of $V$ into $L_{n}\left(U, U_{1}\right)$, such that $g_{v}$ is injective for all nonzero $v$ in $V$, and

$$
f(u \otimes v)=g_{v}(u) \otimes v_{1},
$$

for all $u \in U, v \in V$.

(iii) $\mu$ can be extended to an automorphism $\sigma$ of $D$, and there exist injective $\sigma$-semilinear maps $g: U \rightarrow U_{1}, h: V \rightarrow V_{1}$, such that

$$
f(u \otimes v)=g(u) \otimes h(v),
$$

for all $u \in U, v \in V$.

(iv) $\mu$ can be extended to an anti-automorphism $\sigma$ of $D$, and there exist injective $\sigma$-semilinear maps $g: V \rightarrow U_{1}, h: U \rightarrow V_{1}$, such that

$$
f(u \otimes v)=g(v) \otimes h(u),
$$

for all $u \in U, v \in V$.

Most of the rest of this section is devoted to proving this theorem. We remark that the condition that rank 1 elements are mapped on rank 1 elements implies that $f$ is injective on any rank 1 subgroup of $U \otimes V$. However, $f$ may not be injective on the whole of $U \otimes V$.

The following is an immediate consequence of Lemma 2.

LEMma 3. If $u \in U^{\sharp}$, then $f(u \otimes V) \subseteq u_{1} \otimes V_{1}$, some $u_{1} \in U_{1}^{\sharp}$, or $f(u \otimes V) \subseteq U_{1} \otimes v_{1}$, some $v_{1} \in V_{1}^{\sharp}$.

The next result shows that one of the two possibilities for $f(u \otimes V)$ holds uniformly as $u$ varies over $U^{\sharp}$.

LEMMA 4. It is impossible to have

$$
f(u \otimes V) \leqq u_{1} \otimes V_{1}, f\left(u^{\prime} \otimes V\right) \subseteq U_{1} \otimes v_{1},
$$

where $u, u^{\prime} \in U^{\sharp}, u_{1} \in U_{1}^{\sharp}, v_{1} \in V_{1}^{\sharp}$.

Proof. If $v \in V^{\sharp}$, then $f(u \otimes v)$ and $f\left(u^{\prime} \otimes v\right)$ have rank 1, while $f(u \otimes v)+f\left(u^{\prime} \otimes v\right)=f\left(\left(u+u^{\prime}\right) \otimes v\right)$ has rank 1 or is 0 . By Lemma 1, either $U_{1}\left(f\left(u^{\prime} \otimes v\right)\right)=U_{1}(f(u \otimes v))=\left\langle u_{1}\right\rangle$, or $V_{1}(f(u \otimes$ $v))=V_{1}\left(f\left(u^{\prime} \otimes v\right)\right)=\left\langle v_{1}\right\rangle$. Thus either $f(u \otimes v)$ or $f\left(u^{\prime} \otimes v\right)$ lies in 
$\left(u_{1} \otimes V_{1}\right) \cap\left(U_{1} \otimes v_{1}\right) . \quad$ As a vector space over $k,\left(u_{1} \otimes V_{1}\right) \cap\left(U_{1} \otimes v_{1}\right)$ has dimension equal to the dimension [D:k] of $D$ over $k$, while $V$ has dimension $[D: k] \operatorname{dim}_{D} V$. Hence the inverse image of $\left(u_{1} \otimes V_{1}\right) \cap$ $\left(U_{1} \otimes v_{1}\right)$ under the injective semilinear map $v \rightarrow f(u \otimes v)$ of $V$ into $u_{1} \otimes V_{1}$ is a proper subspace of $V$, and the inverse image of $\left(u_{1} \otimes\right.$ $\left.V_{1}\right) \cap\left(U_{1} \otimes v_{1}\right)$ under the map $v \rightarrow f\left(u^{\prime} \otimes v\right)$ of $V$ into $U_{1} \otimes v_{1}$ is a proper subspace of $V$. Thus $V$ is a union of two proper subspaces, and this is impossible.

We thus have two possibilities:

(A) For every $u \in U$, there exists $u_{1} \in U_{1}$ such that

$$
f(u \otimes V) \cong u_{1} \otimes V_{1} .
$$

(B) For every $u \in U$, there exists $v_{1} \in V_{1}$ such that

$$
f(u \otimes V) \cong U_{1} \otimes v_{1} .
$$

Similarly, we also have two possibilities for the $f(U \otimes v)$ :

(a) For every $v \in V$, there exists $v_{1} \in V_{1}$ such that

$$
f(U \otimes v) \cong U_{1} \otimes v_{1} .
$$

(b) For every $v \in V$, there exists $u_{1} \in U_{1}$ such that

$$
f(U \otimes v) \subseteq u_{1} \otimes V_{1} .
$$

In all we therefore have four cases, $(\mathrm{Aa}),(\mathrm{Ab}),(\mathrm{Ba}),(\mathrm{Bb})$, where (Aa) means that both (A) and (a) hold, etc. We consider these one at a time.

Case (Ab). Fix $v \in V^{\sharp}$ and $u_{1} \in U_{1}^{\sharp}$ satisfying (b). Considering $f(u \otimes v)$, we see that this fixed $u_{1}$ may be used in (A), for every $u \in U$, so that

$$
f(u \otimes v)=u_{1} \otimes h_{u}(v),
$$

where $h_{u}$ is a map of $V$ into $V_{1}$, depending on $u$. The map $h_{u}$ is uniquely determined by $u$ if $u \neq 0$, and we take $h_{0}=0$.

Since the map $v \rightarrow u \otimes v$ is $k$-linear and $f$ is $\mu$-semilinear, $h_{u}$ is also $\mu$-semilinear. If $u$ and $v$ are nonzero, $f(u \otimes v)$ is nonzero, so that $h_{u}(v) \neq 0$. Thus $h_{u}$ is injective if $u \neq 0$. Clearly $h_{u+u^{\prime}}=h_{u}+$ $h_{u^{\prime}}$. Finally, if $\alpha \in D$,

$$
u_{1} \otimes h_{u}(\alpha v)=f(u \otimes \alpha v)=f(u \alpha \otimes v)=u_{1} \otimes h_{u \alpha}(v),
$$

so that $h_{u \alpha}=h_{u} \alpha$. Thus the map $u \rightarrow h_{u}$ is $D$-linear, and case (i) of Theorem A holds.

Case (Ba). An exactly similar argument shows that case (ii) of 
Theorem A holds.

Case (Aa). There exist maps $g_{1}: U \rightarrow U_{1}, h_{1}: V \rightarrow V_{1}, \gamma: U \times V \rightarrow$ $D$, such that

$$
f(u \otimes v)=g_{1}(u) \otimes \gamma(u, v) h_{1}(v)
$$

Since $f$ preserves rank 1 elements, $\gamma(u, v)$ is nonzero when $u$ and $v$ are nonzero.

We prove that, if $u, u^{\prime} \in U^{\sharp}$, and $v, v^{\prime} \in V^{\sharp}$, then

$$
\gamma(u, v)=\gamma\left(u, v^{\prime}\right) \gamma\left(u^{\prime}, v^{\prime}\right)^{-1} \gamma\left(u^{\prime}, v\right) .
$$

There are two cases. First suppose that $h_{1}(v)$ and $h_{1}\left(v^{\prime}\right)$ are linearly independent. From the equations

$$
\begin{aligned}
& f\left(u \otimes\left(v+v^{\prime}\right)\right)=f(u \otimes v)+f\left(u \otimes v^{\prime}\right), \\
& f\left(u^{\prime} \otimes\left(v+v^{\prime}\right)\right)=f\left(u^{\prime} \otimes v\right)+f\left(u^{\prime} \otimes v^{\prime}\right),
\end{aligned}
$$

we have the relations

$$
\begin{aligned}
& \gamma\left(u, v+v^{\prime}\right) h_{1}\left(v+v^{\prime}\right)=\gamma(u, v) h_{1}(v)+\gamma\left(u, v^{\prime}\right) h_{1}\left(v^{\prime}\right), \\
& \gamma\left(u^{\prime}, v+v^{\prime}\right) h_{1}\left(v+v^{\prime}\right)=\gamma\left(u^{\prime}, v\right) h_{1}(v)+\gamma\left(u^{\prime}, v^{\prime}\right) h_{1}\left(v^{\prime}\right) .
\end{aligned}
$$

Since $h_{1}(v)$ and $h_{1}\left(v^{\prime}\right)$ are linearly independent, we have

$$
\gamma(u, v)=\lambda \gamma\left(u^{\prime}, v\right), \gamma\left(u, v^{\prime}\right)=\lambda \gamma\left(u^{\prime}, v^{\prime}\right),
$$

where $\lambda=\gamma\left(u, v+v^{\prime}\right) \gamma\left(u^{\prime}, v+v^{\prime}\right)^{-1}$. The equation (*) follows.

Now suppose that $h_{1}(v)$ and $h_{1}\left(v^{\prime}\right)$ are linearly dependent. Since (B) does not hold, there exists $v^{\prime \prime}$ in $V$ such that $h_{1}\left(v^{\prime \prime}\right)$ and $h_{1}(v)$ are linearly independent. By what we have proved,

$$
\begin{aligned}
& \gamma(u, v)=\gamma\left(u, v^{\prime \prime}\right) \gamma\left(u^{\prime}, v^{\prime \prime}\right)^{-1} \gamma\left(u^{\prime}, v\right), \\
& \gamma\left(u, v^{\prime}\right)=\gamma\left(u, v^{\prime \prime}\right) \gamma\left(u^{\prime}, v^{\prime \prime}\right)^{-1} \gamma\left(u^{\prime}, v^{\prime}\right),
\end{aligned}
$$

and the equation $(*)$ follows.

We now fix $u^{\prime} \in U^{\sharp}, v^{\prime} \in V^{\sharp}$, and set

$$
\begin{aligned}
& g(u)=g_{1}(u) \gamma\left(u, v^{\prime}\right), \text { when } u \in U^{*}, \\
& g(0)=0, \\
& h(v)=\gamma\left(u^{\prime}, v^{\prime}\right)^{-1} \gamma\left(u^{\prime}, v\right) h_{1}(v), \text { when } v \in V^{*}, \\
& h(0)=0 .
\end{aligned}
$$

By the equation $(*)$ we have

$$
f(u \otimes v)=g(u) \otimes h(v),
$$

for all $u \in U, v \in V$. 
If $u \neq 0, v \neq 0$, then $f(u \otimes v) \neq 0$, and so $g(u) \neq 0, h(v) \neq 0$. Since $f$ is $\mu$-semilinear, we see that $g$ and $h$ are $\mu$-semilinear as maps on $U$ and $V$ regarded as vector spaces over $k$. Thus $g$ and $h$ are injective.

If $\alpha \in D, u \in U^{\sharp}, v \in V^{\sharp}$, then

$$
g(u) \otimes h(\alpha v)=f(u \otimes \alpha v)=f(u \alpha \otimes v)=g(u \alpha) \otimes h(v) .
$$

It follows that

$$
\begin{aligned}
& g(u \alpha)=g(u) \bar{\alpha}, \\
& h(\alpha v)=\bar{\alpha} h(v),
\end{aligned}
$$

where $\bar{\alpha} \in D$. The first of these two equations shows that $\bar{\alpha}$ is independent of $v$, and the second shows that it is independent of $u$. If $\alpha, \beta \in D$, then

$$
g(u) \overline{\alpha \beta}=g(u \alpha \beta)=g(u \alpha) \bar{\beta}=g(u) \bar{\alpha} \bar{\beta},
$$

so that $\overline{\alpha \beta}=\bar{\alpha} \bar{\beta}$. Similarly, $\overline{\alpha+\beta}=\bar{\alpha}+\bar{\beta}$. Hence the map $\alpha \rightarrow$ $\bar{\alpha}$ is an endomorphism $\sigma$ of $D$, which clearly extends the automorphism $\ell$ of $k$. Thus $\sigma$ is injective, and, since $D$ is finite-dimensional over $k, \sigma$ is an automorphism of $D$, with $g$ and $h$ both $\sigma$-semilinear. Thus case (iii) of Theorem A holds.

Case $(\mathrm{Bb})$. Here case (iv) of Theorem $\mathrm{A}$ holds. The argument is essentially the same as for case (Aa), and is omitted.

This completes the proof of Theorem A.

We remark that there are conditions under which cases (i) and (ii) of Theorem A cannot occur, for example when $U=U_{1}, V=V_{1}$, $U$ and $V$ are finite-dimensional, and $k$ is algebraically closed, since then $L_{u}(V, V)$ and $L_{\mu}(U, U)$ do not contain any subspace of dimension greater than 1, whose nonzero elements are all injective [8]. Also we mention that a converse to Theorem $\mathrm{A}$ is easily formulated and proved.

A similar theorem to Theorem A was proved by Jacob [6], in which $D$ is any division ring with more than 2 elements, and the semilinearity condition on $f$ is replaced by the assumption that $f$ is bijective and that both $f$ and its inverse satisfy a condition of "coherence invariance."

2. Maps on spaces of linear transformations. If $D$ is a division ring, $V$ is a left vector space over $D$ and $V^{\prime}$ is a right vector space over $D$, then $\left(V, V^{\prime}\right)$ is called a pair of dual vector spaces if there is given a nondegenerate bilinear form $b: V \times V^{\prime} \rightarrow$ 
$D\left[7\right.$, p. 69]. Usually $b\left(v, v^{\prime}\right)$ is abbreviated $\left(v, v^{\prime}\right)$. A Hausdorff topology can then be defined to make $V$ a topological group, by taking the annihilators $Y^{\perp}$ in $V$ of finite-dimensional subspaces $Y$ of $V^{\prime}$ as a fundamental set of neighborhoods of 0 . This is called the $V^{\prime}$-topology on $V$, and can also be described by the fact that the closure of any subspace $W$ of $V$ is the double annihilator $W^{\perp \perp}$; in particular, finite-dimensional subspaces are closed [7, p. 71]. Similarly, there is a $V$-topology defined on $V^{\prime}$.

Suppose $\left(U, U^{\prime}\right)$ and $\left(V, V^{\prime}\right)$ are two pairs of dual vector spaces over $D$. If $x: U \rightarrow V$ is semilinear with respect to an automorphism $\sigma$ of $D$, a $\sigma^{-1}$-semilinear map $x^{\prime}: V^{\prime} \rightarrow U^{\prime}$ is called the adjoint of $x$ if

$$
\left(x(u), v^{\prime}\right)=\left(u, x^{\prime}\left(v^{\prime}\right)\right)^{\sigma},
$$

for all $u \in U, v^{\prime} \in V^{\prime}$. If $U$ and $V$ are given the $U^{\prime}$-topology and $V^{\prime}$-topology, then $x$ has an adjoint if and only if it is continuous. Similar remarks hold for $\sigma$-semilinear maps of $U$ into $V^{\prime}$, where $\sigma$ is an anti-automorphism of $D$, etc.

The continuous linear maps from $U$ to $V$ form an additive group $L(U, V)$ (more accurately, $L_{U^{\prime}, V^{\prime}}(U, V)$ ). An element $x$ of $L(U, V)$ is finite-valued if the image $x(U)$ is a finite-dimensional subspace of $V$, and the finite-valued maps in $L(U, V)$ form a subgroup $F(U, V)$. This subgroup can be identified with the tensor product $U^{\prime} \otimes V$, so that elements $u^{\prime} \otimes v$ of rank 1 in $U^{\prime} \otimes V$ are identified with maps $u \rightarrow\left(u, u^{\prime}\right) v$, which are precisely the continuous linear maps $x$ for which $x(U)$ has dimension 1 (that is, $x$ has rank 1 in the usual sense for linear maps).

The set $V^{U}$ of all mappings of $U$ into $V$ has the product topology obtained from the $V^{\prime}$-topology of $V$. As a subset of $V^{U}$, $L(U, V)$ inherits this topology, and becomes a topological group, in which a fundamental set of neighborhoods of 0 consists of the subgroups

$$
N(W, X)=\left\{x \in L(U, V) \mid x(W) \subseteq X^{\perp}\right\},
$$

where $W$ and $X$ range over the finite-dimensional subspaces of $U$ and $V^{\prime}$ respectively. We call this the symmetric topology on $L(U, V)$, since the map $x \rightarrow x^{\prime}$ (adjoint of $x$ ) is a homeomorphism of $L(U, V)$ on $L\left(V^{\prime}, U^{\prime}\right)$, taken with its similarly defined topology. The symmetric topology is coarser than the finite topology usually defined on $L(U, V)$ [7, p. 29]. $F(U, V)$ is a dense subgroup of $L(U, V)$.

If $U$ and $V$ are finite-dimensional, all the topologies mentioned are discrete, and then all maps are of course continuous. 
If $D$ is a division algebra over a field $k$, identified with a subfield of the center of $D$, then $L(U, V)$ is a vector space over $k$, and $F(U, V)$ is a subspace. The identification of $F(U, V)$ with $U^{\prime} \otimes V$ is a vector space isomorphism. In this case, every finitedimensional subspace of $L(U, V)$ is closed. In $\S 3$ we shall use a more general result.

LEMMA 5. Let $B$ and $C$ be subspaces of $L(U, V)$ such that $B \cong$ $C$ and $\operatorname{dim}_{k} C / B=n<\infty$.

(i) If $B$ is closed, then $C$ is also closed.

(ii) In any case, if $\bar{B}$ and $\bar{C}$ are the closures of $B$ and $C$, $\operatorname{dim}_{k} \bar{C} / \bar{B} \leqq n$.

Proof. (i) By induction, we need only consider the case $n=1$, so that $C=B+k x$, where $x \notin B$. Since $B$ is closed, there exist finite-dimensional subspaces $W_{1}, X_{1}$ of $U, V^{\prime}$, such that $x+N\left(W_{1}\right.$, $\left.X_{1}\right)$ is disjoint from $B$. This implies that $N\left(W_{1}, X_{1}\right)$ contains no element of $C$ outside $B$.

Let $y$ be an element of the closure of $C$. Then $y+N\left(W_{1}, X_{1}\right)$ meets $C$, so that $y-a x-b \in N\left(W_{1}, X_{1}\right)$, where $a \in k, b \in B$. If $W$, $X$ are finite-dimensional subspaces of $U, V^{\prime}$ containing $W_{1}, X_{1}$ respectively, we find that $y-a^{\prime} x-b^{\prime} \in N(W, X)$, where $a^{\prime} \in k, b^{\prime} \in B$. Since $N(W, X) \subseteq N\left(W_{1}, X_{1}\right)$, we see that $N\left(W_{1}, X_{1}\right)$ contains ( $a-$ $\left.a^{\prime}\right) x+b-b^{\prime}$, and thus $a=a^{\prime}$. Hence $(y-a x)+N(W, X)$ meets $B$. Since the $N(W, X)$ with $W \supseteqq W_{1}$ and $X \supseteqq X_{1}$ form a base for the system of neighborhoods of 0 , and $B$ is closed, it follows that $y$ $a x \in B$, so that $y \in C$.

(ii) Since $\operatorname{dim}_{k}(\bar{B}+C) / \bar{B}=\operatorname{dim}_{k} C /(\bar{B} \cap C) \leqq n$, (i) implies that $\bar{B}+C=\bar{C}$, and the result follows.

We now consider the case that $D$ is a finite-dimensional division algebra over $k$. In the next result, continuity is always taken with respect to the topologies we have defined. In particular, $L(U, V)$ (and its subspaces) are given the symmetric topology. If $x, y$ are maps, the product $x y$ is the composition " $y$ followed by $x$."

TheOREM B. Let $\left(U, U^{\prime}\right),\left(V, V^{\prime}\right),\left(U_{1}, U_{1}^{\prime}\right),\left(V_{1}, V_{1}^{\prime}\right)$ be pairs of dual vector spaces over $D$, where $D$ is a finite-dimensional division algebra over a field $k$, and suppose $\operatorname{dim}_{D} U>1, \operatorname{dim}_{D} V>1$. Let $A$ be a subspace of $L(U, V)$ containing $F(U, V)$, and let

$$
f: A \longrightarrow L\left(U_{1}, V_{1}\right)
$$

be a continuous map which is semilinear with respect to an automorphism $\mu$ of $k$. If $f$ maps elements of rank 1 in $A$ on elements 
of rank 1 in $L\left(U_{1}, V_{1}\right)$, then one of the following holds.

(i) There exist a nonzero element $u_{1}^{\prime}$ of $U_{1}^{\prime}$ and a continuous $\mu$-semilinear map $h: A \rightarrow V_{1}$ whose kernel contains no element of rank 1, such that

$$
f(x)=u_{1}^{\prime} \otimes h(x),
$$

for all $x \in A$.

(ii) There exist a nonzero element $v_{1}$ of $V_{1}$ and a continuous $\mu$-semilinear map $g: A \rightarrow U_{1}^{\prime}$ whose kernel contains no element of rank 1, such that

$$
f(x)=g(x) \otimes v_{1}
$$

for all $x \in A$.

(iii) $\mu$ can be extended to an automorphism $\sigma$ of $D$, and there exist a continuous injective $\sigma$-semilinear map $h: V \rightarrow V_{1}$ and $a$ continuous $\sigma^{-1}$-semilinear map $g: U_{1} \rightarrow U$ whose image $g\left(U_{1}\right)$ is dense in $U$, such that

$$
f(x)=h x g,
$$

for all $x \in A$.

(iv) $\mu$ can be extended to an anti-automorphism $\sigma$ of $D$, and there exist a continuous injective $\sigma$-semilinear map $h: U^{\prime} \rightarrow V_{1}$ and a continuous $\sigma^{-1}$-semilinear map $g: U_{1} \rightarrow V^{\prime}$ whose image $g\left(U_{1}\right)$ is dense in $V^{\prime}$, such that

$$
f(x)=h x^{\prime} g,
$$

for all $x \in A$, where $x^{\prime}$ is the adjoint of $x$.

Proof. Since $F(U, V)$ and $F\left(U_{1}, V_{1}\right)$ are spanned by their elements of rank 1, the restriction of $f$ maps $F(U, V)$ into $F\left(U_{1}, V_{1}\right)$. Since $F(U, V)=U^{\prime} \otimes V, F\left(U_{1}, V_{1}\right)=U_{1}^{\prime} \otimes V_{1}$, we can apply Theorem A.

Suppose case (i) of Theorem A holds. Then there exists a nonzero element $u_{1}^{\prime}$ of $U_{1}^{\prime}$ such that

$$
f(F(U, V)) \subseteq u_{1}^{\prime} \otimes V_{1} .
$$

Suppose $x_{1} \in L\left(U_{1}, V_{1}\right), x_{1} \notin u_{1}^{\prime} \otimes V_{1}$. Then the kernel of $x_{1}$ does not contain the annihilator $H_{1}$ of $u_{1}^{\prime}$ in $U_{1}$, so that there exists a onedimensional subspace $W_{1}$ of $H_{1}$ such that $x_{1}\left(W_{1}\right) \neq 0$. There exists a one-dimensional subspace $X_{1}$ of $V_{1}^{\prime}$ such that $x_{1}\left(W_{1}\right)$ is not contained in the annihilator $X_{1}^{\perp}$ of $X_{1}$. If $y_{1} \in x_{1}+N\left(W_{1}, X_{1}\right)$, then $y_{1}\left(W_{1}\right)$ is not contained in $X_{1}^{\prime}$, so that $y_{1} \notin u_{1}^{\prime} \otimes V_{1}$. Hence $u_{1}^{\prime} \otimes V_{1}$ is a closed subset of $L\left(U_{1}, V_{1}\right)$. Since $f$ is continuous and $F(U, V)$ is dense in 
$A$, we have $f(A) \leqq u_{1}^{\prime} \otimes V_{1}$, so that

$$
f(x)=u_{1}^{\prime} \otimes h(x),
$$

for all $x \in A$, where $h$ is a map of $A$ into $V_{1}$. Since $f$ is $\mu$-semilinear and nonzero on elements of rank 1 , so is $h$.

Suppose $X_{1}$ is any finite-dimensional subspace of $V_{1}^{\prime}$. Since $u_{1}^{\prime} \neq$ 0 , there exists a one-dimensional subspace $W_{1}$ of $U_{1}$ which is not in the annihilator of $u_{1}^{\prime}$. By continuity of $f$, there exists a neighborhood $N$ of 0 in $A$, such that $f(N) \subseteq N\left(W_{1}, X_{1}\right)$, so that $u_{1}^{\prime} \otimes h(x)$ maps $W_{1}$ into $X_{1}^{\perp}$, for all $x \in N$. Hence $h(N) \subseteq X_{1}^{\perp}$. Thus $h$ is continuous, and case (i) of Theorem $B$ holds.

If case (ii) of Theorem A holds, a similar argument shows that assertion (ii) of Theorem B holds.

Next suppose case (iii) of Theorem A holds, so that $\mu$ can be extended to an automorphism $\sigma$ of $D$, and there exist injective $\sigma$ semilinear maps $g^{\prime}: U^{\prime} \rightarrow U_{1}^{\prime}, h: V \rightarrow V_{1}$, such that

$$
f\left(u^{\prime} \otimes v\right)=g^{\prime}\left(u^{\prime}\right) \otimes h(v),
$$

for all $u^{\prime} \in U^{\prime}, v \in V$. Suppose $W_{1}$ is a finite-dimensional subspace of $U_{1}$. Let $v$ be a nonzero element of $V$, so that $h(v) \neq 0$, and there exists a one-dimensional subspace $X_{1}$ of $V_{1}^{\prime}$ which does not annihilate $h(v)$. Since $f$ is continuous, there exist finite-dimensional subspaces $W, X$ of $U, V^{\prime}$, such that $f(A \cap N(W, X)) \subseteq N\left(W_{1}, X_{1}\right)$. If $u^{\prime} \in W^{\perp}$, then $u^{\prime} \otimes v \in A \cap N(W, X)$, so that $g^{\prime}\left(u^{\prime}\right) \otimes h(v) \in N\left(W_{1}\right.$, $\left.X_{1}\right)$. Since $h(v) \notin X_{1}^{\perp}, g^{\prime}\left(u^{\prime}\right) \in W_{1}^{\perp}$. This shows that $g^{\prime}$ is continuous. Similarly, $h$ is continuous.

Since $g^{\prime}$ is continuous, it possesses an adjoint map $g: U_{1} \rightarrow U$, which is a continuous $\sigma^{-1}$-semilinear map. If $u^{\prime} \in U^{\prime}, v \in V, u \in U$, then $g^{\prime}\left(u^{\prime}\right) \otimes h(v)$ maps $u$ on the element

$$
\left(u, g^{\prime}\left(u^{\prime}\right)\right) h(v)=\left(g(u), u^{\prime}\right)^{o} h(v)=h\left(\left(g(u), u^{\prime}\right) v\right),
$$

and $\left(g(u), u^{\prime}\right) v$ is the image of $g(u)$ under $u^{\prime} \otimes v$. Hence,

$$
f(x)=h x g,
$$

for all elements $x$ of rank 1, and hence for all $x$ in $F(U, V)$.

Now define a map $f_{1}$ on the whole of $A$ by setting $f_{1}(x)=h x g$, $x \in A$. Since $g$ and $h$ are continuous, $f_{1}$ maps $A$ into $L\left(U_{1}, V_{1}\right)$. If $W_{1}, X_{1}$ are finite-dimensional subspaces of $U_{1}, V_{1}^{\prime}$, then $g\left(W_{1}\right)$ is a finite-dimensional subspace $W$ of $U$, and there exists a finite-dimensional subspace $X$ of $V^{\prime}$ such that $h\left(X^{\perp}\right) \subseteq X_{1}^{\perp}$, since $h$ is continuous. Clearly $f_{1}(A \cap N(W, X)) \subseteq N\left(W_{1}, X_{1}\right)$, so that $f_{1}$ is continuous. Since $L\left(U_{1}, V_{1}\right)$ is Hausdorff and $f$ and $f_{1}$ agree on the dense subset $F(U, V)$ of $A$, it follows that $f=f_{1}$, so that $f(x)=h x g$, for all 
$x \in A$.

Since $g^{\prime}$ is injective, the definition of the adjoint map shows that the only vector in $U^{\prime}$ annihilating $g\left(U_{1}\right)$ is 0 . Thus the closure of $g\left(U_{1}\right)$ in $U$ is $g\left(U_{1}\right)^{\perp \perp}=U$, so that $g\left(U_{1}\right)$ is dense in $U$. Assertion (iii) of Theorem $B$ holds.

If case (iv) of Theorem A holds, a similar argument shows that assertion (iv) of Theorem B holds. This completes the proof of Theorem B.

As in Theorem A, a converse to Theorem B is easily formulated and proved.

3. Maps on primitive algebras. An associative algebra $A$ over a field $k$ is said to be primitive if it possesses a faithful irreducible right module. If such an algebra has a minimal one-sided ideal, then it has both minimal left ideals and minimal right ideals, and also has a faithful irreducible left module [7, Chap. IV]. In this case $A$ has, to within isomorphism, only one faithful irreducible right module. The commuting algebra of this module is a division algebra $D$, which can be realized within $A$ as the subalgebra $e A e$, where $e$ is an idempotent generating a minimal right ideal. By the structure theorem for primitive algebras with minimal one-sided ideals, there is a pair of dual vector spaces $\left(V, V^{\prime}\right)$ over $D$ such that $F(V, V) \subseteq A \leqq L(V, V)$. We take $V$ with the $V^{\prime}$-topology, and $A$ with the topology induced from the symmetric topology on $L(V, V)$. If $A$ is not a division algebra, then $\operatorname{dim}_{D} V>1$.

THeOREM C. Let $D$ be a finite-dimensional division algebra over a field $k$, and $\left(V, V^{\prime}\right)$ a pair of dual vector spaces over $D$, with $\operatorname{dim}_{D} V>1$. Let $A$ be a subalgebra of $L(V, V)$ containing $F(V, V)$, and let $f$ be a continuous map of $A$ into itself which is semilinear with respect to an automorphism $\mu$ of $k$, such that $f$ preserves zero products. Assume

(i) The kernel of $f$ does not contain any elements of rank 1 in $F(V, V)$.

(2) The image of $f$ is dense in $A$, or $V$ is finite-dimensional and the kernel of $f$ does not contain any divisors of zero.

Then $\mu$ can be extended to an automorphism $\sigma$ of $D$, and there exist a $\sigma$-semilinear homeomorphism $h: V \rightarrow V$ and a nonzero element $\alpha$ of the center of $D$, such that

$$
f(x)=h x d_{\alpha} h^{-1},
$$

for all $x \in A$, where $d_{\alpha}$ is the homothety $v \rightarrow \alpha v$ on $V$ defined by $\alpha$. 
Proof. We shall show that $f$ preserves elements of rank 1, so that Theorem $\mathrm{B}$ can be applied. For $x \in A$, form the left and right annihilators

$$
\begin{aligned}
& L(x)=\{y \in A \mid y x=0\}, \\
& R(x)=\{z \in A \mid x z=0\},
\end{aligned}
$$

subspaces of $A$.

Suppose that $x$ is of rank 1 , and let $v$ be a nonzero element of the image $x(V)$. Consider the $k$-linear map $\theta: A \rightarrow V /$ ker $x$ given by $\theta(y)=\overline{y(v)}$, the coset of $y(v)(\bmod \operatorname{ker} x)$. Clearly $\theta$ is surjective. If $y \in \operatorname{ker} \theta$, let $z=u^{\prime} \otimes y(v)$, where $u^{\prime}$ is an element of $V^{\prime}$ such that $\left(v, u^{\prime}\right)=1$. Then $z \in R(x)$, since $y(v) \in \operatorname{ker} x$, and $y-z \in L(x)$, since $y(v)=z(v)$. Hence $\operatorname{ker} \theta \subseteq L(x)+R(x)$, so that

$$
\operatorname{dim}_{k} A /(L(x)+R(x)) \leqq \operatorname{dim}_{k} A / \operatorname{ker} \theta=\operatorname{dim}_{k} V / \operatorname{ker} x=[D: k] .
$$

(Actually $\operatorname{ker} \theta=L(x)+R(x)$, so that equality holds.)

Next suppose that $x$ has rank greater than 1 . Let $v, w$ be linearly independent vectors in $x(V)$. Since $\operatorname{ker} x$ is closed, $V / \operatorname{ker} x$ has a Hausdorff quotient topology. The $k$-linear map $\theta: A \rightarrow V /$ ker $x \oplus V / \operatorname{ker} x$ given by

$$
\theta(y)=\overline{y(v)} \oplus \overline{y(w)}
$$

is continuous and surjective. If $y \in L(x), z \in R(x)$, then $y(v)=y(w)=$ $0, z(v) \in \operatorname{ker} x$, and $z(w) \in \operatorname{ker} x$, so that $y+z \in \operatorname{ker} \theta$. Since $\operatorname{ker} \theta$ is closed, we see that $\operatorname{dim}_{k} A / \operatorname{cl}(L(x)+R(x)) \geqq \operatorname{dim}_{k} A / \operatorname{ker} \theta=2 \operatorname{dim}_{k} V /$ ker $x \geqq 4[D: k]$, where cl denotes closure in the symmetric topology.

If $V$ is finite-dimensional, then $A$ is the algebra of all linear transformations on $V$, and $y \in R(x)$ if and only if $y$ maps $V$ into $\operatorname{ker} x$, so that $R(x)$ is isomorphic as a $k$-vector space to the direct sum of $\operatorname{dim}_{D} V$ copies of $\operatorname{ker} x$, so that

$$
\operatorname{dim}_{k} R(x)=[D: k]\left(\operatorname{dim}_{D} V\right)\left(\operatorname{dim}_{D} \operatorname{ker} x\right),
$$

and $\operatorname{dim}_{k} R(x)$ is a strictly decreasing function of rank $x$.

Since $f$ preserves zero products, $f(L(x)) \subseteq L(f(x))$ and $f(R(x)) \subseteq$ $R(f(x))$. If $x$ has rank 1 , then

$$
\operatorname{dim}_{k} f(A) / f(L(x)+R(x)) \leqq \operatorname{dim}_{k} A /(L(x)+R(x)) \leqq[D: k] .
$$

If the image $f(A)$ is dense in $A$, we see by Lemma 5 that

$$
\operatorname{dim}_{k} A / \operatorname{cl}(L(f(x))+R(f(x))) \leqq \operatorname{dim}_{k} A / \operatorname{cl}(f(L(x)+R(x))) \leqq[D: k] .
$$

It follows that $f(x)$ has rank at most 1 . Since $f(x) \neq 0$ by condition (1), $f(x)$ has rank 1 . If the second alternative of condition (2) holds, then 


$$
\operatorname{dim}_{k} R(f(x)) \geqq \operatorname{dim}_{k} f(R(x))=\operatorname{dim}_{k} R(x),
$$

so that rank $f(x) \leqq \operatorname{rank} x$. Again we see that $f(x)$ has rank 1 .

We can now apply Theorem B. Suppose case (i) holds, so that there is a nonzero element $u^{\prime}$ of $V^{\prime}$ such that $f(A) \cong u^{\prime} \otimes V$. In the proof of Theorem B we saw that $u^{\prime} \otimes V$ is closed in $L(V, V)$. If $f$ has dense image, we see that

$$
F(V, V) \subseteq A \subseteq u^{\prime} \otimes V
$$

so that $\operatorname{dim} V=1$, a contradiction. If $V$ is finite-dimensional, let $v^{\prime}$ be a nonzero element of $V^{\prime}$. There is an injective $\mu$-semilinear map $h\left(h_{v^{\prime}}\right.$ in the notation of Theorem A) of $V$ into itself, such that $f\left(v^{\prime} \otimes v\right)=u^{\prime} \otimes h(v)$, for all $v \in V$. Since $V$ is finite-dimensional, $h$ is surjective. Choose $v$ so that $\left(h(v), u^{\prime}\right) \neq 0$. Let $w^{\prime}$ be a nonzero element of the annihilator of $v$ in $V^{\prime}$, and $w$ any nonzero element of $V$. Then

$$
\left(w^{\prime} \otimes w\right)\left(v^{\prime} \otimes v\right)=0, f\left(w^{\prime} \otimes w\right) f\left(v^{\prime} \otimes v\right) \neq 0,
$$

a contradiction. Hence, case (i) does not hold.

A similar argument shows that case (ii) does not hold.

Suppose now that case (iii) of Theorem B holds. Then $\mu$ extends to an automorphism $\sigma$ of $D$, and there exist a continuous injective $\sigma$-semilinear map $h: V \rightarrow V$ and a continuous $\sigma^{-1}$-semilinear map $g$ : $V \rightarrow V$ with dense image, such that

$$
f(x)=h x g,
$$

for all $x \in A$. Suppose the image of $f$ is dense in $A$. If $v$ is a nonzero element of $V$, choose $y \in A$ such that $y(v) \neq 0$. Let $W$ be the subspace of $V$ spanned by $v$, and choose a one-dimensional subspace $X$ of $V^{\prime}$ such that $y(v) \notin X^{\perp}$. There exists $x \in A$ such that $f(x) \in y+N(W, X)$, so that $f(x)(v)-y(v) \in X^{\perp}$. Hence $f(x)(v) \neq 0$, so that $v \notin \operatorname{ker} g$. Hence $g$ is injective. If $V$ is finite-dimensional, then $g$ is surjective and hence again $g$ is injective.

Let $W$ be a one-dimensional subspace of $V$ and $X$ any onedimensional subspace of $W^{\perp}$. We can take rank 1 elements $x, y$ in $A$ such that $y(V)=W$ and $\operatorname{ker} x=X^{\perp}$. Then $x y=0$, so that

$$
h x g h y g=f(x) f(y)=0 .
$$

Since $g$ has dense image and $h$ is injective, $x g h y=0$, so that $g(h(W)) \leqq X^{\perp}$. Since $W$ is closed, we have $W=W^{\perp \perp}$, the intersection of all the subspaces $X^{\perp}$ as $X$ ranges over the one-dimensional subspaces of $W^{\perp}$. Hence,

$$
g(h(W)) \subseteq W
$$


for every one-dimensional subspace $W$ of $V$. It follows that $g h$ is a homothety $d_{\alpha}: v \rightarrow \alpha v$, where $\alpha$ is an element of $D$. Since $g h$ is $D$-linear, $\alpha$ must lie in the center of $D$. Since $g h$ is injective, $\alpha \neq 0$, so that $d_{\alpha}$ is a homeomorphism of $V$ on itself. Thus $g$ is bijective, and hence $h$ is also bijective. Since $h^{-1}=d_{\alpha}^{-1} g$ is continuous, $h$ is a homeomorphism. Setting $g=d_{\alpha} h^{-1}$, we obtain the desired result.

Finally consider the case (iv) of Theorem B. We have a continuous injective semilinear map $h: V^{\prime} \rightarrow V$ and a continuous semilinear map $g: V \rightarrow V^{\prime}$ with dense image, such that

$$
f(x)=h x^{\prime} g,
$$

for all $x \in A$. As before, we see that $g$ is injective. Let $W$ be a one-dimensional subspace of $V$, and $y$ an element of $A$ having image $W$. Since $\operatorname{dim}_{D} V>1$, we can choose a nonzero element $v^{\prime}$ of $W^{\perp}$. Since $y^{\prime} \neq 0$, and $g$ and $h$ are injective, there exists an element $v \in V$ which is not annihilated by $g\left(h\left(y^{\prime}\left(V^{\prime}\right)\right)\right)$. Let $x=v^{\prime} \otimes v$. Then, since $\operatorname{ker} x^{\prime}$ annihilates $x(V)$, we see that $x^{\prime} g h y^{\prime} \neq 0$. Since $g$ has dense image and $h$ is injective, $f(x) f(y)=h x^{\prime} g h y^{\prime} g \neq 0$. However, $x y=0$. Thus this case does not occur, and Theorem $\mathrm{C}$ is proved.

Our result can be stated in intrinsic terms, that is, without reference to the representation of $A$ as an algebra of linear transformations. In the situation of the theorem, $F(V, V)$ is the socle soc $(A)$, which is the sum of the minimal right ideals of $A$, or equivalently the sum of the minimal left ideals of $A$. (Also $\operatorname{soc}(A)$ is the unique minimal ideal of $A$.) The finite-dimensional subspaces of $V$ are just the images of the elements of $F(V, V)$, and the annihilators in $V$ of finite-dimensional subspaces of $V^{\prime}$ are just the kernels of the elements of $F(V, V)$. Hence the symmetric topology on $A$ is determined intrinsically by taking as base for the neighborhoods of 0 the sets $\{y \in A \mid x y w=0\}$, where $w$ and $x$ range over soc $(A)$. The elements of rank 1 are characterized as the elements generating minimal right (or left) ideals.

If $h$ and $\alpha$ are as in the conclusion of the theorem, the map $x \rightarrow x d_{\alpha}$ is an element of the centroid (center) of $L(V, V)$, and the map $x \rightarrow h x h^{-1}$ is a semilinear automorphism of $L(V, V)$. If $A \neq$ $L(V, V)$, it is not clear that these maps leave $A$ invariant, except in the case that $A=F(V, V)$. To avoid complications, we give an intrinsic restatement of Theorem $\mathrm{C}$ only for the case that $A$ is simple.

COROLlaRy D. Let $A$ be a simple associative algebra over a field $k$, which is not a division algebra, and suppose that $A$ contains a nonzero idempotent $e$ such that eAe is finite-dimensional over $k$. 
Then $A$ is a topological group with a base for the neighborhoods of 0 being given by the sets $\{y \in A \mid x y w=0\}$, where $x, w \in A$. Let $f$ be a continuous semilinear map on $A$ which preserves zero products, such that $f$ is injective on every minimal right ideal of $A$. Assume that either the image of $f$ is dense in $A$, or $A$ is finite-dimensional and the kernel of $f$ does not contain any divisors of zero. Then $f$ is the product of an element of the unit group of the centroid of $A$ with a semilinear automorphism of $A$.

Proof. Since a nilpotent one-sided ideal generates a nilpotent two-sided ideal, simplicity implies that $A$ has no nonzero nilpotent one-sided ideals. Let $e$ be a nonzero idempotent for which the dimension of $e A e$ is as small as possible. If $b$ is a nonzero element of $e A e$, so that $b=e b=b e$, we see that

$$
(b e A e)^{n} b A=(b A)^{n+1} \neq 0,
$$

so that $(b e A e)^{n} \neq 0$. Hence, $e A e$ has no nilpotent right ideals. Let $J$ be a minimal right ideal of $e A e$. Then, $J=f e A e$, where $f$ is an idempotent in $e A e[7, \mathrm{p}$. 57]. Since $f=f e$, we have

$$
f A f=f e A f e \subseteq f e A e=J .
$$

The minimality of the dimension of $e A e$ implies that $f A f=e A e$, so that $J=e A e$. Thus $e A e$ has no proper nonzero right ideals. Similarly, $e A e$ has no proper nonzero left ideals. It follows that $e A e$ is a division algebra, and hence that $e A$ is a minimal right ideal of $A$ [7, p. 65]. Using the structure theorem for primitive rings with minimal one-sided ideals, we are now in a position to apply Theorem C, which immediately gives Corollary D.

We remark that when $D=k$ in Theorem $\mathrm{C}$, if $A$ is finite-dimensional and $f$ is assumed to be bijective, then the determination of $f$ is immediate from a result of Dieudonné [4]. In another direction, if $f$ is assumed to be a homeomorphism and both $f$ and $f^{-1}$ preserve zero products, then $f$ and $f^{-1}$ preserves elements of rank 1 , which are characterized as those elements $x$ whose right annihilators $R(x)$ are maximal in the set of all right annihilators of nonzero elements of $A$. Then $f$ can be determined by using the theorem of Jacob [6], without any assumption on the dimension of $D$.

4. Examples. Maps preserving zero products which do not satisfy the conditions of Theorem $\mathrm{C}$ can be constructed in a number of ways. Take $V$ and $A$ as in Theorem C.

(a) If $W$ is a closed subspace of $V$, then the set 


$$
A_{W}=\{x \in A \mid x(V) \subseteq W, x(W)=0\}
$$

is a closed subalgebra of $A$ in which all products are zero. Any continuous semilinear map $f$ of $A$ into $A_{W}$ will preserve zero products, but will not satisfy condition (2) of Theorem C. If $V$ is infinite-dimensional, it is possible for $f$ to be injective, so that condition (1) is satisfied. For example, we may have $V=W \oplus U, V^{\prime}=$ $U^{\star} \oplus W^{\perp}$, where $W$ and $U$ are each linearly homeomorphic with $V$. Let $g: U \rightarrow V, h: W \rightarrow V$ be linear homeomorphisms. If $x \in A=$ $L(V, V)$, then $h^{-1} x g$ is a continuous linear map of $U$ into $W$, which extends to an element $f(x)$ of $A_{W}$. The map $f$ is a $k$-linear homeomorphism of $A$ on $A_{w}$.

(b) Any algebra endomorphism of $A$ will of course also preserve zero products. In the infinite-dimensional case, it is possible to have nonzero endomorphisms of $A$ which are not automorphisms. Suppose $W$ is a subspace of $V$ such that there exists a linear homeomorphism $h: W \rightarrow V$. If $x \in L(V, V)$, we obtain a continuous linear transformation $h^{-1} x h$ on $W$. If $h$ can be extended to a continuous linear map on $V$, then $h^{-1} x h$ extends to an element of $L(V, V)$, and we obtain a continuous injective endomorphism of $L(V, V)$. The closure of the image of this endomorphism consists of linear transformations mapping $V$ into the closure of $W$. If $W$ is not dense in $V$, then the image is not dense in $L(V, V)$, so that again condition (2) is not satisfied.

(c) In another direction, suppose $T$ is a dense subspace of $V$. We note that if $y$ is a continuous finite-valued linear transformation on $T$, then the kernel of $y$ is open in $T$, since it has finite condimension, so that there exists a finite-dimensional subspace $X$ of $V^{\prime}$ such that $y\left(T \cap X^{\perp}\right)=0$. It follows from this fact, and the denseness of $T$, that if $v$ is any element of $V$, then $y\left(T \cap\left(v+X^{\perp}\right)\right)$ has a single element $w$. Further, $\{w\}$ is the intersection of all the sets $y\left(T \cap\left(v+Y^{\perp}\right)\right)$, as $Y$ ranges over all finite-dimensional subspaces of $V^{\prime}$. It is easily checked that the map $v \rightarrow w$ is the unique extension of $y$ to an element of $F(V, V)$. Now, if there exists a linear homeomorphism $s: T \rightarrow V$, then every element $x$ of $F(V, V)$ defines a finite-valued linear transformation $s^{-1} x s$ on $T$, which can be uniquely extended to an element of $F(V, V)$. This gives an injective endomorphism of $F(V, V)$ with dense image, which is not continuous if $T$ is a proper subspace of $V$.

(d) Let $V$ have countably infinite basis $v_{1}, v_{2}, v_{3}, \cdots$, and $V^{\prime}$ the dual basis $v_{1}^{\prime}, v_{2}^{\prime}, v_{3}^{\prime}, \cdots$, such that $\left(v_{i}, v_{j}^{\prime}\right)=\delta_{i j}$. All of the situations (a), (b), (c) may be exhibited by taking $W$ to be generated by $v_{1}, v_{3}, v_{5}, \cdots, U$ by $v_{2}, v_{4}, v_{6}, \cdots$, and $T$ by $v_{1}-v_{2}, v_{2}-v_{3}, v_{3}-v_{4}, \cdots$.

(e) It seems to be open whether there exists a continuous 
semilinear map preserving zero products which satisfies condition (2) but not condition (1) in Theorem C.

\section{REFERENCES}

1. P. Botta, Linear maps that preserve singular and nonsingular matrices, Linear Algebra and Appl., 20 (1978), 45-49.

2. E. P. Botta and S. Pierce, The preservers of any orthogonal group, Pacific J. Math., 70 (1977), 37-49.

3. J. Dieudonné, La Géométrie des Groupes Classiques, Springer-Verlag, Berlin, 1955.

4. - Sur une généralisation du groupe orthogonal à quatre variables, Arch. Math., 1 (1949), 282-287.

5. J. D. Dixon, Rigid embedding of simple groups in the general linear group, Canad. J. Math., 29 (1977), 384-391.

6. H. G. Jacob, Coherence invariant mappings on Kronecker products, Amer. J. Math., 77 (1955), 177-189.

7. N. Jacobson, Structure of rings, Amer. Math. Soc. Colloquium Publ., 27 (1956).

8. M. Marcus and B. N. Moyls, Transformations on tensor product spaces, Pacific J. Math., 9 (1959), 1215-1221.

9. W. Watkins, Linear maps that preserve commuting pairs of matrices, Linear Algebra and Appl., 14 (1976), 29-35.

10. W. J. Wong, Maps on simple algebras preserving zero products. II: Lie algebras of linear type.

Received May 31, 1978. Research partially supported by National Science Foundation grant MCS 77-02057 at the University of Notre Dame.

UNIVERSITY OF NOTRE DAME

Notre DAME, IN 46556 



\section{PACIFIC JOURNAL OF MATHEMATICS}

\section{EDITORS}

DONALD BABBITT (Managing Editor)

University of Galifornia

Los Angeles, California 90024

Hugo RossI

University of Utah

Salt Lake City, UT 84112

C. C. MoOre AND ANDrew OGG

University of California

Berkeley, CA 94720

\section{J. DugundjI}

Department of Mathematics University of Southern California

Los Angeles, California 90007

R. Finn and J. Milgram

Stanford University

Stanford, California 94305

ASSOCIATE EDITORS

E. F. Beckenbachi

B. H. NeumanN

F. WOLF

K. YOSHIDA

\section{SUPPORTING INSTITUTIONS}

UNIVERSITY OF BRITISH COLUMBIA

CALIFORNIA INSTITUTE OF TECHNOLOGY

UNIVERSITY OF CALIFORNIA

MONTANA STATE UNIVERSITY

UNIVERSITY OF NEVADA, RENO

NEW MEXICO STATE UNIVERSITY

OREGON STATE UNIVERSITY

UNIVERSITY OF OREGON
UNIVERSITY OF SOUTHERN CALIFONIA

STANFORD UNIVERSITY

UNIVERSITY OF HAWAII

UNIVERSITY OF TOKYO

UNIVERSITY OF UTAH

WASHINGTON STATE UNIVERSITY

UNIVERSITY OF WASHINGTON

Printed in Japan by International Academic Printing Co., Ltd., Tokyo, Japan 


\section{Pacific Journal of Mathematics \\ Vol. 89 , No. 1 \\ May, 1980}

David Bressoud, A note on gap-frequency partitions ................ 1

John David Brillhart, A double inversion formula ................. 7

Frank Richard Deutsch, Günther Nürnberger and Ivan Singer, Weak

Chebyshev subspaces and alternation .......................

Edward Richard Fadell, The relationship between Ljusternik-Schnirelman

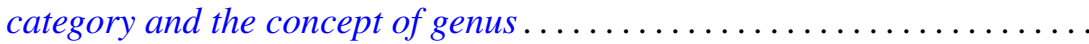

Harriet Jane Fell, On the zeros of convex combinations of polynomials.......

John Albert Fridy, An addendum to: "Tauberian theorems via block

dominated matrices" ..................................

Andrzej Granas, Ronald Bernard Guenther and John Walter Lee, Applications of topological transversality to differential equations. I. Some nonlinear

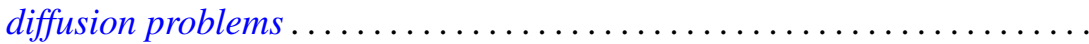

David E. Handelman and G. Renault, Actions of finite groups on self-injective

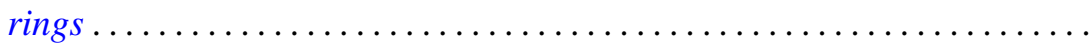

Michael Frank Hutchinson, Local $\Lambda$ sets for profinite groups .............

Arnold Samuel Kas, On the handlebody decomposition associated to a

Lefschetz fibration...

Hans Keller, On the lattice of all closed subspaces of a Hermitian space.....

P. S. Kenderov, Dense strong continuity of pointwise continuous

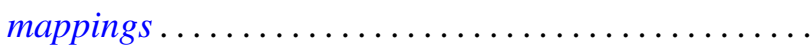

Robert Edward Kennedy, Krull rings.................

Jean Ann Larson, Richard Joseph Laver and George Frank McNulty,

Square-free and cube-free colorings of the ordinals ...

Viktor Losert and Harald Rindler, Cyclic vectors for $L^{p}(G)$

John Rowlay Martin and Edward D. Tymchatyn, Fixed point sets of

1-dimensional Peano continua...

Augusto Nobile, On equisingular families of isolated singularities ...

Kenneth Joseph Prevot, Imbedding smooth involutions in trivial bundles ...

Thomas Munro Price, Spanning surfaces for projective planes in four space.

Dave Riffelmacher, Sweedler's two-cocycles and Hochschild cohomology....

Niels Schwartz, Archimedean lattice-ordered fields that are algebraic over

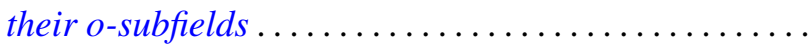

Chao-Liang Shen, A note on the automorphism groups of simple dimension groups.

Kenneth Barry Stolarsky, Mapping properties, growth, and uniqueness of

Vieta (infinite cosine) products ...

Warren James Wong, Maps on simple algebras preserving zero products. I.

The associative case ............................. 